\title{
A Case of Delayed Onset Nephrogenic Systemic Fibrosis After Gadolinium Based Contrast Injection
}

\author{
Jong Geol Do, M.D., Young Bum Kim, M.D., Dae Gu Lee, M.D., Ji Hye Hwang, M.D.
}

Department of Physical and Rehabilitation Medicine, Samsung Medical Center, Sungkyunkwan University School of Medicine, Seoul 135-710, ${ }^{1}$ Department of Rehabilitation Medicine, Pusan National University Yangsan Hospital, Yangsan 602-739, Korea

Nephrogenic systemic fibrosis (NSF) is a rare fibrosing disorder of the skin and joints that occurs in patients with advanced renal insufficiency. This condition is progressive and can be seriously disabling. Gadolinium based contrast agent (GBCA) has been identified as a potential cause of this condition. A 56-years-old man in hemodialysis developed stiffness and contracture of the whole limbs eight years after frequent GBCA exposure for cervical magnetic resonance imaging. For the first time in Korea, we report late-onset nephrogenic systemic fibrosis after GBCA exposure and performed an electrophysiologic study of this condition.

Key Words Nephrogenic systemic fibrosis, Gadolinium, Electrophysiologic study

\section{INTRODUCTION}

Nephrogenic systemic fibrosis (NSF) is a systemic sclerosis or scleroderma-like fibrosing disorder of the skin that develops in patients with renal insufficiency. It presents with progressive hardening or induration of the skin of the extremities. Renal failure patients who undergo magnetic resonance imaging (MRI) using a gadolinium-

Received July 27, 2011; Accepted February 13, 2012

Corresponding author: Ji Hye Hwang

Department of Physical and Rehabilitation Medicine, Samsung Medical Center, Sungkyunkwan University School of Medicine, 50, Ilwon-dong, Gangnam-gu, Seoul 135-710, Korea

Tel: +82-2-3410-2848, Fax: +82-2-3410-2820, E-mail: hwanglee@skku.edu (c) This is an open-access article distributed under the terms of the Creative Commons Attribution Non-Commercial License (http:// creativecommons.org/licenses/by-nc/3.0) which permits unrestricted noncommercial use, distribution, and reproduction in any medium, provided the original work is properly cited.

Copyright $\odot 2012$ by Korean Academy of Rehabilitation Medicine based contrast agent (GBCA) have been reported to be at a higher risk for developing this disease. Therefore, the U.S. Food and Drug Administration has recommended that GBCA be carefully used in MRI for patients with a glomerular filtration rate (GFR) of less than $30 \mathrm{ml} /$ $\mathrm{min} / 1.73 \mathrm{~m}^{2}$ or those receiving dialysis. The U.S. and European countries also recommend that such patients receive hemodialysis within three hours after exposure to GBCA. Since scleromyxedema was first reported in 14 chronic hemodialysis patients in 2000 , over 300 cases have been reported since and recently two cases were reported in Korea. ${ }^{1,2}$ The two cases involved an acute occurrence of NSF after MRI was performed using GBCA. The authors of this study reported a case of delayed onset NSF in a chronic renal failure patient after use of a GBCA. For the first time in Korea, we report late-onset nephrogenic systemic fibrosis after GBCA exposure and performed an electrophysiologic study of this condition. 


\section{CASE REPORT}

A 56-year-old man visited our hospital with complaints of bilateral limb weakness, joint contractures of both upper and lower limbs, and whole body skin fibrosis. He had been diagnosed with chronic renal failure of an unknown cause ten years before and was recommended to undergo dialysis, which was not conducted because he refused the treatment. Nine years ago, an arteriovenous shunt was performed after emergency dialysis through catheterization of the internal jugular vein due to serious uremia accompanied by edema and fatigue. Neck pain and redness occurred on the area where the arteriovenous shunt was inserted and a cervical MRI showed an extradural abscess in the fifth and sixth cervical vertebrae. The abscess was then removed and a discectomy was performed at the C-5 and C- 6 level. Despite the patient was renal insufficient, he underwent 5 cervical MRIs over the course of a year. Thereafter, he started peritoneal dialysis but due to recurrent peritonitis, he began to receive hemodialysis six years ago. Five months before his visit to our hospital, he displayed symptoms of edema of the bilateral lower limbs together with skin thickening of the bilateral upper and lower extremities. Contractures of the shoulders, wrists, hip joints, and knee and ankle joints then gradually occurred. He developed skin nodules between the first and second digit and had a gait disability due to weakness of the bilateral lower limbs and contracture of the bilateral knees. He denied any family history of such diseases other than that his mother was diagnosed with diabetes mellitus.

In regards to his vital signs after hospitalization, his blood pressure, pulse rate, body temperature, height, and weight were $120 / 70 \mathrm{mmHg}, 70$ beats per minute, $36.5^{\circ} \mathrm{C}$, $170 \mathrm{~cm}$ and $65 \mathrm{~kg}$, respectively. He appeared chronic ill looking. According to his complete blood count test, his hemoglobin was $10.7 \mathrm{~g} / \mathrm{dl}$, white blood cell count was $6,160 / \mathrm{mm}^{3}$, eosinophil was $5.8 \%$, blood nitrogen level was $34.6 \mathrm{mg} / \mathrm{dl}$, blood creatinine level was $5.81 \mathrm{mg} / \mathrm{dl}$ (estimated GFR at $10.1 \mathrm{ml} / \mathrm{min} / 1.73 \mathrm{~m}^{2}$ ), sodium was $139 \mathrm{mEq} / \mathrm{L}$, potassium was $4.8 \mathrm{mEq} / \mathrm{L}$, calcium was 11.8 $\mathrm{mEq} / \mathrm{L}$, phosphorus was $6.5 \mathrm{mg} / \mathrm{dl}, \mathrm{C}$-reactive protein was $0.92 \mathrm{mg} / \mathrm{dl}$, erythrocyte sedimentation rate was 62 $\mathrm{mm} / \mathrm{hr}$, creatine kinase was $49 \mathrm{IU} / \mathrm{L}$, lactate dehydrogenase was $370 \mathrm{IU} / \mathrm{L}$, parathyroid hormone was $332.9 \mathrm{pg} /$ $\mathrm{m}$, and B2-microglobulin level was $23.3 \mathrm{mg} / \mathrm{dl}$. In the rheumatoid test, he was fluorescent antinuclear antibody positive $(1: 80)$. However, the results for the anti-RNP, anti-Sm, anti-SSA (Ro), anti-SSB (La), anti-Scl-70, anticentromere antibody and anti-Jo tests were negative. The values obtained from his protein immunoassay and thyroid function test were within the normal limit range (Table 1). Electrocardiography showed a right bundle branch block and echocardiography found a slight mitral valve regurgitation, mild pulmonary arterial hypertension, and bilateral cardiomegaly. The simple chest radiography indicated no abnormal finding in the pulmonary parenchyma and the pulmonary function was found to be normal.

Three months after development of symptoms, an electrophysiologic study was performed. Although no abnormal findings were observed in the nerve conduction study regarding the bilateral lower extremities and the right upper extremity, needle electromyography indicated positive sharp waves and a fibrillation potential in the bilateral vastus medialis, gastrocnemius, tibialis anterior, iliopsoas, and right peroneus longus. However, no abnormal spontaneous activity was noted in the bilateral first dorsal interosseous, biceps brachi, and left peroneus longus. Decreased motor unit action potentials (MUAP) were observed in the left gastrocnemius and tibialis anterior. Short-duration MUAPs were observed in the left vastus medialis, tibialis anterior, and iliopsoas and short duration polyphasic MUAPs in the right vastus medialis, gastrocnemius, and peroneus longus. With regard to muscle recruitment patterns, early recruitment was observed in the right tibialis anterior and reduced recruitment in the bilateral brachial biceps, left gastrocnemius, peroneus longus, right vastus medialis, iliopsoas, and

Table 1. Laboratory Findings

\begin{tabular}{ll}
\hline Eosinophilia & $5.8 \%$ \\
Paraproteinemia & No \\
ANA titer/pattern & $1: 80 /$ homogeneous \\
\hline Anti-centromere/Scl-70 & $\mathrm{N} / \mathrm{N}$ \\
\hline Anti-RNP/Anti-Sm & $\mathrm{N} / \mathrm{N}$ \\
\hline Anti-Ro/Anti-La/Anti-Jo & $\mathrm{N} / \mathrm{N} / \mathrm{N}$ \\
ESR & $62 \mathrm{~mm} / \mathrm{hr}$ \\
CRP & $0.92 \mathrm{mg} / \mathrm{dl}$ \\
CK & $\mathrm{CK} 49 \mathrm{IU} / \mathrm{L}$ \\
\hline
\end{tabular}

ANA: Anti-nuclear antibody, ESR: Erythrocyte sedimentation rate, CRP: C-reactive protein, CK: Creatine kinase, $\mathrm{N}$ : Negative 
Table 2. Results of the Needle Electromyography

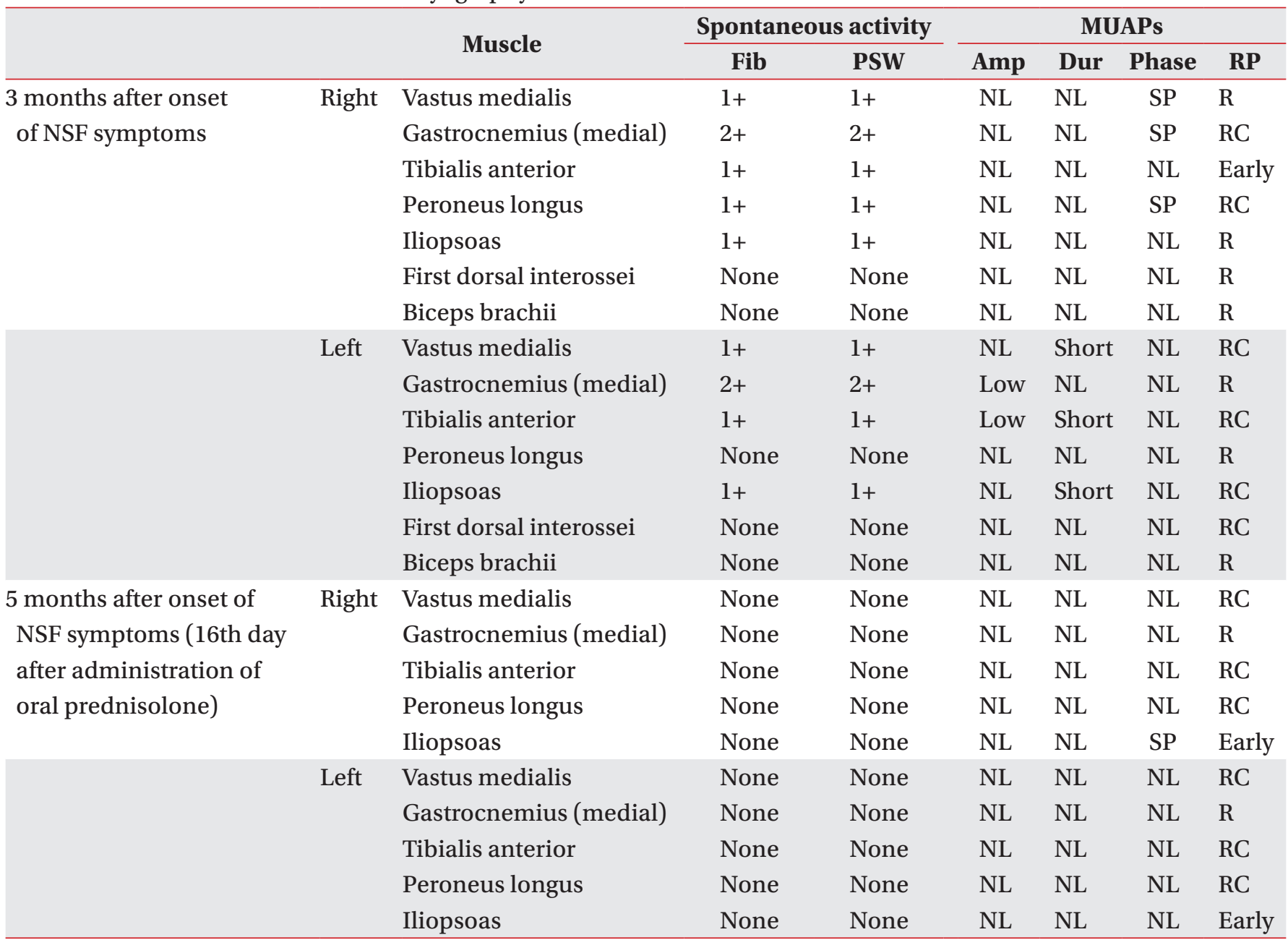

MUAP: Motor unit action potential, Fib: Fibrillation, PSW: Positive sharp wave, Amp: Amplitude, Dur: Duration, RP: Recruitment pattern, NSF: Nephrogenic systemic fibrosis, NL: Normal, SP: Short polyphasic, R: Reduced, RC: Reduced to complete

first dorsal interosseous (Table 2).

Histopathologic examination of the skin, fascia, and muscle tissues in the posterior right calf was performed. Histopathologic examination revealed dermal fibrosis with collagen deposition in the dermis, and septal fibrosis in the subcutaneous tissue (Fig. 1). Non-enhanced MRI of the bilateral lower limbs showed muscular atrophy in the bilateral gastrocnemius and an increased signal intensity in the fascia along the edge of the soleus muscle (Fig. 2). He was diagnosed as NSF. Although, the patient was administered $30 \mathrm{mg}$ of prednisolone twice daily, his symptoms did not improve.

On the thirteenth day after admission, he was transferred to the department of physical and rehabilitation medicine to treat his gait disability, weakness of the bilat- eral lower extremities, and joint contractures of the whole limbs. He had pain whenever he moved his shoulders, elbows, hip joints, knees, and ankles, with numerical rating scale at 3 at rest and 7 in motion. During sleeping he woke up about ten times due to pain. Physical examination indicated whole body skin fibrosis and skin nodules between the first and second fingers. A manual muscle test showed that the bilateral lower limb muscles had a muscle strength grade of 4 , except for the bilateral upper limb and bilateral hip joint flexor and hip extensor muscles. The bilateral upper limb and bilateral hip joint flexor and hip extensor muscles grades were 3+. A Gower's sign was also observed. Hypesthesia was not noted in a sensory examination. Limit of motion was observed in all joints of the upper and lower limbs. Knee Flexion 

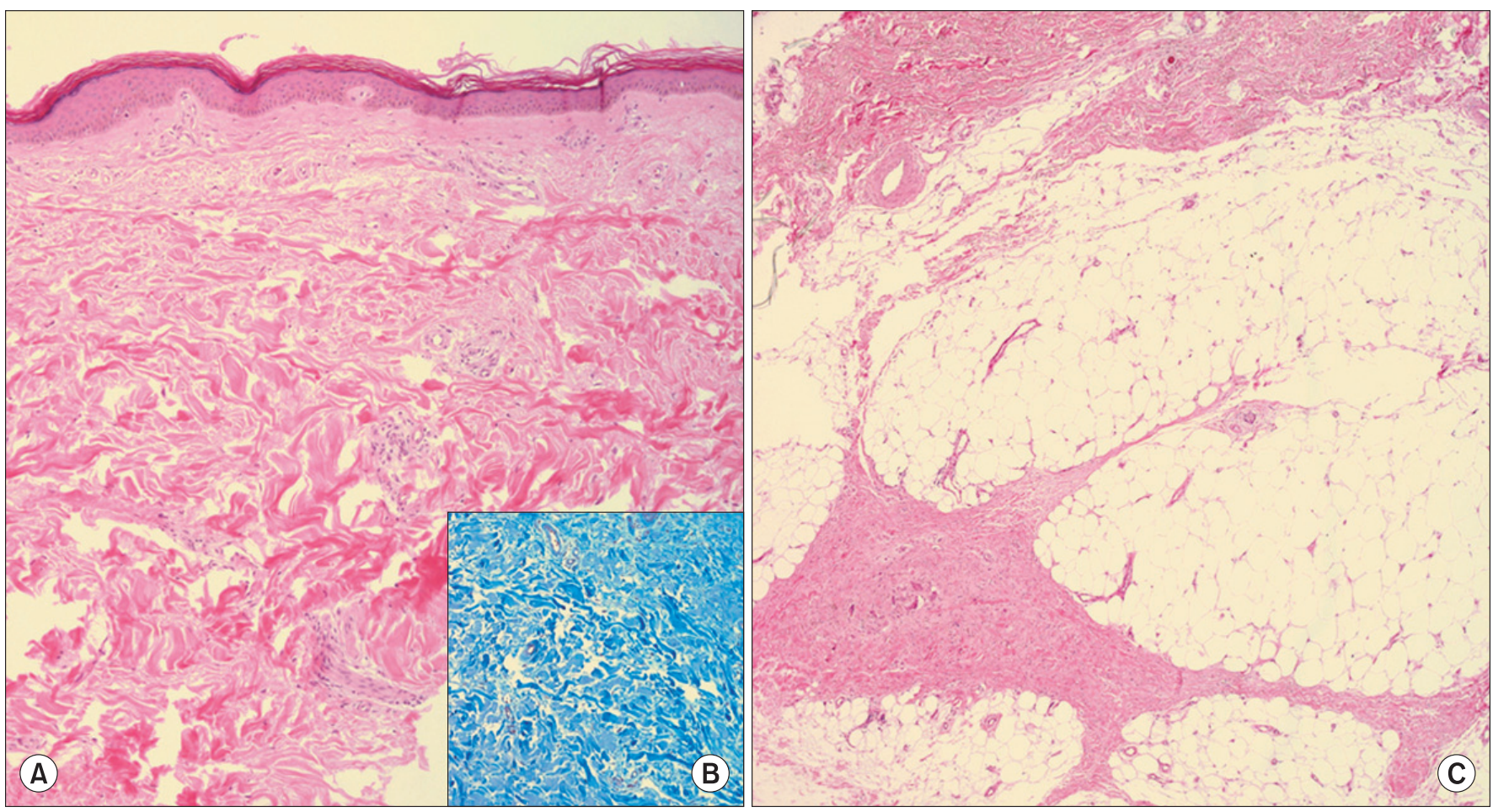

Fig. 1. Histopathologic findings of nephrogenic systemic fibrosis. (A) Dermal fibrosis with thick collagen deposition (H\&E stain, $\times 100$ ), (B) Increased dermal collagen of the papillary dermis and deep dermis (Masson's Trichrome stain, $\times 200$ ), (C) Septal fibrosis of subcutaneous adipose tissue (H\&E stain, $\times 100)$.
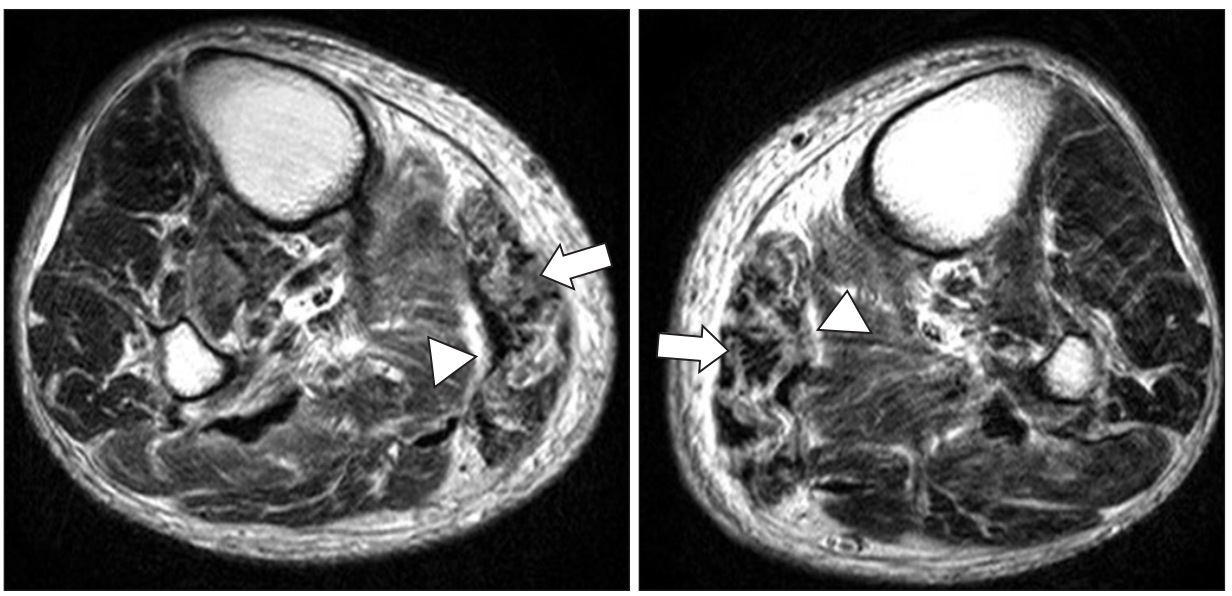

Fig. 2. Lower extremity non-contrast MRI in patient with nephrogenic systemic fibrosis (T2weighted image). Fatty atrophic changes in both gastrocnemius medial head (arrows), high signal intensity throughout the fascia (arrowheads).

contractures were 15 degrees for the right and left sides and ankle plantar flexion contractures were 25 degrees in the right side and 20 degrees in the left side (Fig. 3). Due to weakness of the bilateral lower limbs, bilateral knee flexion contractures, and ankle plantar flexion contractures, he was not able to perform an independent gait. He required moderate assistance from others when washing his face, maintaining personal hygiene, and putting on clothes because of flexion contractures of the bilateral shoulder and elbow joints. There was no abnormal finding in the deep tendon reflex, Babinski reflex, and Hoffman's reflex tests.

Four months after he developed symptoms, he was prescribed prednisolone. After sixteen days on the medication, an electrophysiologic study revealed no abnormal spontaneous activity in the bilateral vastus medialis, gastrocnemius, tibialis anterior, iliopsoas, and right peroneus longus. In the electromyography analysis, no 

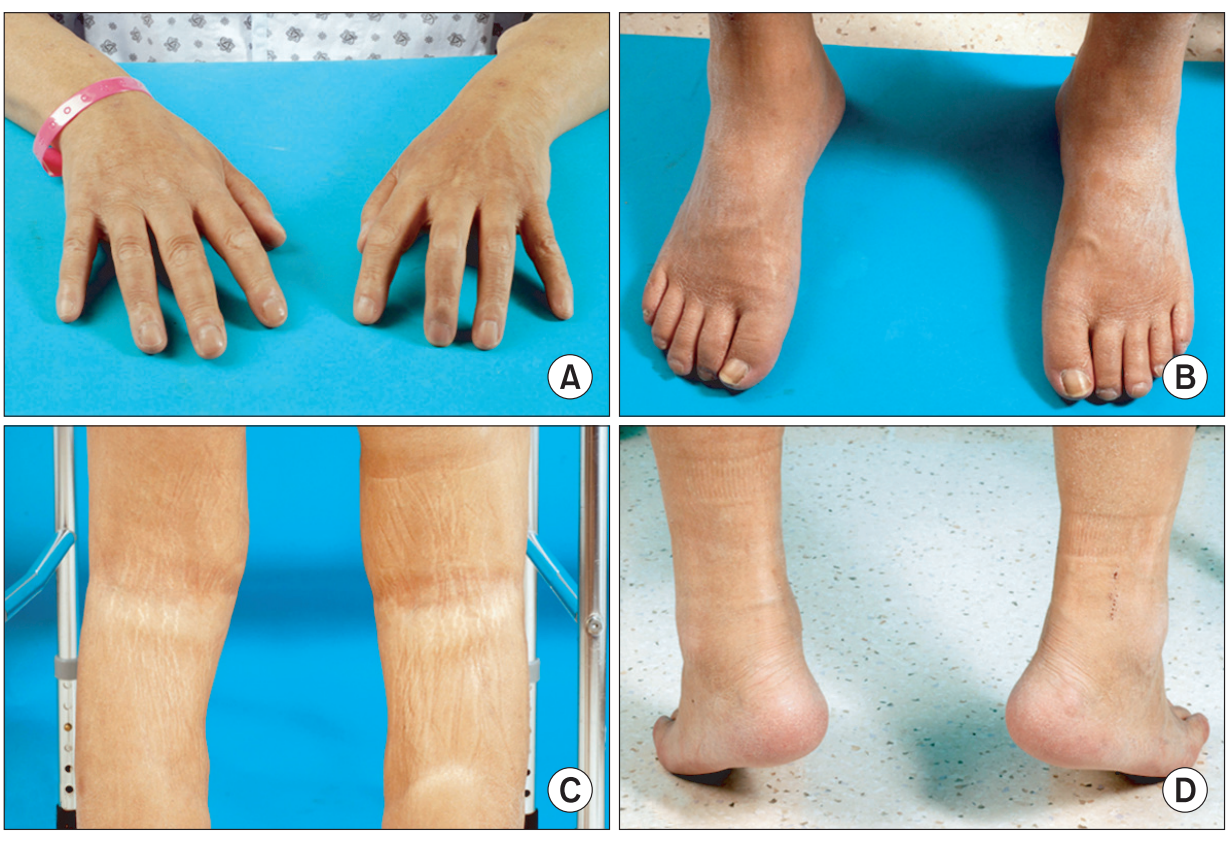

Fig. 3. Clinical findings of the nephrogenic systemic fibrosis patient. (A) Diffuse thickening and hyperpigmentation of skin in both hands (B) Thickening and fibrotic induration of the skin in both feet (C) Both knee flexion contracture and lack of hair on the skin (D) Both ankle plantar contracture.

myopathy-like findings in the motor unit action potential were observed, except for in the right iliopsoas. Regarding the right iliopsoas, short duration polyphasic MUAPs and early recruitment pattern MUAPs were noted. Left peroneus longus, iliopsoas, right vastus medialis, and iliopsoas showed an increased MUAPs recruitment pattern relative to a previous study (Table 2).

Physical modalities, stretching exercise, and joint mobilization techniques were utilized to treat the joint contractures of the bilateral upper and lower limbs using paraffin on the finger to relieve finger stiffness. Training was performed to improve the activities of daily living in an occupational therapy room. He was also provided physical therapy to enhance bilateral lower limb muscle strength and improve gait function using a walker, together with independent gait training in a hydrotherapy room. During gait movement, his heels could not contact the floor due to his ankle plantar flexion contractures; therefore, outsoles were attached onto the heels so that heel contacted the floor. He received rehabilitation for a month in the department of physical and rehabilitation medicine. After this treatment, the pain in motion was reduced with an NRS of 5, and the issues associated with sleeping due to pain also improved. In addition, his finger stiffness was also alleviated. Because of the increased range of motion of the bilateral shoulder and elbow joints, he required minimal assistance from others in washing his face, maintaining personal hygiene, and putting on clothes. The modified Barthel index, which was originally 51 at the time of transfer to the department of physical and rehabilitation medicine had increased to 77 at discharge from the hospital. In addition, the bilateral lower extremity joint range of motion, bilateral knee flexion contractures and ankle plantar contractures improved by 5 degrees. When he was discharged from hospital, he was able to walk independently using a standard walker.

\section{DISCUSSION}

NSF is a rare disease that causes papule, erythema, edema, and induration of the skin and involves systemic infiltration that affects deep muscles, the pericardium, and diaphragm. Its accurate incidence rate is still not known. NSF is diagnosed based on the history of exposure to GBCA and clinical and pathological findings in patients with decreased renal function. Clinically, skin induration and papule and patterned plaques and joint contractures may occur. Pathologically, an increase in dermal cellularity, thick and thin collagen bundles, septal involvement, osseous metaplasia, and CD34 positive fibroblasts may be observed. NSF should be differentially diagnosed from scleromyxedema, scleroderma, systemic sclerosis, eosinophilic fasciitis, and lipodermatosclerosis. ${ }^{3}$ In the protein immunoassay, eosinophil, and rheumatoid tests of our patient, no abnormal findings were observed; therefore, 
scleromyxedema, eosinophilic fasciitis, and systemic sclerosis were excluded. Lipodermatosclerosis does not involve joint contractures and scleroderma is characterized by a white lesion of the sclerotic plaque. Accordingly, these two diseases were also excluded.

The patient in our case had decreased renal function and was exposed to an excess amount of gadolinium due to the fact that he was subjected to five MRIs over a year. Clinical findings indicated progressive skin induration and papule, and all joint contractures of the bilateral upper and lower limbs. MRI of the bilateral lower extremities found muscular atrophy localized to the bilateral gastrocnemius and an increased signal intensity in the fascia along the edge of the soleus muscle was observed. Pathological analysis indicated skin collagen deposition and fibrosis. Accordingly, he was diagnosed with NSF. ${ }^{3}$

The frequency of NSF is known to differ according to a patient's stage of renal failure and degree of exposure to gadolinium. The incidence of NSF in patient with decreased renal function has been reported to range from 2 to $7 \%$ and exposure to ionic gadolinium is more likely to cause NSF than exposure to nonionic gadolinium. It is known that the risk of developing NSF increases in patients with hyperparathyroidism or hypothyroidism, those who take high doses of erythropoietin, hypercalcemia, hyperphosphatemia, those with a high risk of thrombosis, and patients with metabolic acidosis, and those who receive intravenous injection of iron. ${ }^{4}$ Association between NSF and gadolinium has been known, but a clear understanding of the pathophysiology of this disease is still unknown. It has been shown that gadolinium triggers a dendritic cell response and produces transforming growth factor beta 1 , resulting in tissue fibrosis. Then antigen-presenting B cells cause molecular mimicry and activate autoantibody production, which results in tissue fibrosis. Another theory is that a bone marrow response causes the of release fibrocytes, which accumulate in infiltrated organs, increasing collagen production and resulting in tissue fibrosis. ${ }^{5}$ NSF mostly occurs 2 to 4 weeks after exposure to GBCA but the disease could develop even years after GBCA exposure.$^{6,7}$ It has been reported that delayed onset NSF might be caused by a continuous release of gadolinium from GBCA that has accumulated in the bones. ${ }^{8}$ Unlike the two domestic cases in which NSF-related symptoms occurred within two weeks after exposure to gadolinium, our patient developed NSF eight years later. In regard to our patient, it is possible that the delayed onset NSF may have resulted from gadolinium exposure. He likely developed NSF because his glomerular filtration rate (GFR) was $10 \mathrm{ml} /$ $\min / 1.73 \mathrm{~m}^{2}$, he was exposed to a relatively large amount of gadolinium since he was subjected to 5 MRIs within one year he displayed hyperparathyroidism, hypercalcemia, hyperphosphatemia, and he continuously used erythropietin three times weekly.

In nerve conduction and electromyography analysis, the results typically range from normal to sensory motor polyneuropathy and varied myopathy-like results have often been reported often been reported in patient with NSF. ${ }^{9}$ In our case, the nerve conduction result was normal and abnormal spontaneous activity, short polyphasic MUAPs, early recruitment pattern MUAPs were observed in the electromyography analysis. These findings were similar to the electrophysiologic findings of inflammatory myopathy.

Most NSF progresses into a chronic disease and its mortality rate is $28 \%$. Severe skin symptoms or rapid progression are related to a poor prognosis. However, there is no effective treatment for NSF. As in our case, $5 \%$ of patients who undergo joint contractures have difficulty in gait and activities associated with daily living. About $50 \%$ of NSF patients develop joint contractures. Rehabilitation and walking aids may be helpful in preventing joint contractures and enhancing the activities of daily living and mobility of NSF patients. ${ }^{10}$ Further prevention of joint contractures progression and enhanced activities of daily living performance were observed for our patient through rehabilitation. He also reported that the degree of stiffness and pain resulting from contractures were reduced. In addition, heel outsoles and a standard walker enabled him to perform an independent gait, which contributed to a functional recovery.

When renal failure patients receive MRI, use of gadolinium agents should be reduced. In patients who develop NSF, active rehabilitation treatment aimed at preventing joint contracture and helping functional recovery must be considered.

\section{REFERENCES}

1. Koo TH, Lee DH, Baek HK, Kim DK, Kim BK, Hong $\mathrm{SH}, \mathrm{An}$ WS. A case of nephrogenic systemic fibrosis following gadolinium exposure in a peritoneal dialysis patient. Korean J Med 2010; 78: 507-511 
2. Hong MH, Koo HM, Choi J, Ahn JR, Chon HJ, Kim C, Lee ST. A case of nephrogenic systemic fibrosis after gadolinium-based contrast agent injection. Korean J Med 2010; 78: 127-131

3. Girardi M, Kay J, Elston DM, Leboit PE, Abu-Alfa A, Cowper SE. Nephrogenic systemic fibrosis: clinicopathological definition and workup recommendations. J Am Acad Dermatol 2011; 65: 1095-1106

4. Prince MR, Zhang H, Morris M, MacGregor JL, Grossman ME, Silberzweig J, DeLapaz RL, Lee HJ, Magro $\mathrm{CM}$, Valeri AM. Incidence of nephrogenic systemic fibrosis at two large medical centers. Radiology 2008; 248: 807-816

5. Mendoza FA, Artlett CM, Sandorfi N, Latinis K, PieraVelazquez S, Jimenez SA. Description of 12 cases of nephrogenic fibrosing dermopathy and review of the literature. Semin Arthritis Rheum 2006; 35: 238-249

6. Collidge TA, Thomson PC, Mark PB, Traynor JP, Jardine AG, Morris ST, Simpson K, Roditi GH. Gadolinium-enhanced MR imaging and nephrogenic systemic fibrosis: retrospective study of a renal replacement therapy cohort. Radiology 2007; 245: 168-175

7. Matsumoto Y, Mitsuhashi Y, Monma F, Kawaguchi M, Suzuki T, Miyabe C, Igarashi A, Tsuboi R. Nephrogenic systemic fibrosis: a case report and review on Japanese patients. J Dermatol 2011; 38: 1-5

8. Thakral C, Alhariri J, Abraham JL. Long-term retention of gadolinium in tissues from nephrogenic systemic fibrosis patient after multiple gadoliniumenhanced MRI scans: case report and implications. Contrast Media Mol Imaging 2007; 2: 199-205

9. Levine JM, Taylor RA, Elman LB, Bird SJ, Lavi E, Stolzenberg ED, McGarvey ML, Asbury AK, Jimenez SA. Involvement of skeletal muscle in dialysis-associated systemic fibrosis (nephrogenic fibrosing dermopathy). Muscle Nerve 2004; 30: 569-577

10. Evenepoel P, Zeegers M, Segaert S, Claes K, Kuypers D, Maes B, Flamen P, Fransis S, Vanrenterghem Y. Nephrogenic fibrosing dermopathy: a novel, disabling disorder in patients with renal failure. Nephrol Dial Transplant 2004; 19: 469-473 